\title{
Can You Hear Me Now? Refining the PCMH Model and an Overlooked Disability Affecting Seniors
}

\author{
Nitin Budbwar, MD, FAAFP' \\ Soraya Gollop, $P b D^{2}$ \\ ${ }^{1}$ Chief of Geriatric Medicine, Department of Internal Medicine, University of New Mexico, Albuquerque, New Mexico \\ ${ }^{2}$ Consulting Medical Ethicist
}

Ann Fam Med 2020;18:482-483. https://doi.org/10.1370/afm.2611.

$\mathrm{T}$ The 2019/2020 coronavirus pandemic has shone a spotlight on our medical system and is forcing changes. We stand in a unique moment where we have the opportunity to shape these changes for the better. Our guiding principle for change should be the place where health care costs meet the quality of life that health care can deliver. This guiding principle reflects the "Triple Aim" of health care: improving the experience of care, improving the health of populations, and reducing per capita costs of health care. ${ }^{1}$ Effective primary care is central to meeting these goals, and within this, the treatment of seniors is especially important. Health care costs for seniors are disproportionately high vs the general population, accounting for $34 \%$ of overall medical expenditure. ${ }^{2}$ Preserving the quality of life of individuals as they age is not only good medicine; it is a matter of equity. Access to health care that preserves an individual's abilities to meaningfully engage in community and the activities necessary for daily living can radically impact their quality of life. As Norman Daniels argued, we ought to aim at preserving not only the lifespan of individuals, but the quality of those years lived. ${ }^{3,4}$

In this edition, 2 research papers provide evidence for how we can progress toward the Triple Aim of health care with a focus on senior health, and crucially, how we can improve the quality of life for seniors. Burton et $\mathrm{al}^{5}$ examine the Patient-Centered Medical

Conflicts of interest: author reports none.

\section{CORRESPONDING AUTHOR}

Nitin Budhwar, MD, FAAFP

Department of Internal Medicine

MSC10-5550

1 University of New Mexico

Albuquerque, New Mexico 87131

NBudhwar@salud.unm.edu
Home (PCMH) model with a focus on Medicare beneficiaries to identify which of its myriad requirements are most valuable. Using Medicare claims-based data, they identify 6 PCMH activities that are not only costsaving but lead to better patient outcomes. This is an impressively well-designed study utilizing data from 394 practices in the Centers for Medicare \& Medicaid Services 8-state Multi-Payer Advanced Primary Care Practice Demonstration. They identify 2 activities resulting in significant cost savings, including reduc-

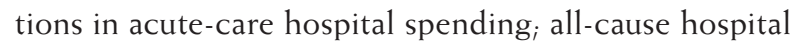
admissions; and rate of emergency department (ED) visits: (1) the use of registries to identify and remind patients due for preventive services (eg, cancer screenings) ${ }_{i}(2)$ using patient registries for pre-visit planning, clinician reminders, patient outreach, and population health monitoring. Four further activities they highlight are associated with other benefits: (3) engaging patients with chronic conditions in goal-setting and action-planning reduces ED visits and hospital admissions $_{i}(4)$ primary care clinicians monitoring patients during hospital stays reduces acute care spending, (5) establishing referral protocols with commonly referred-to clinicians reduces ED visits; and (6) practices with systematic quality improvement approaches also reduce ED visits. To indicate the magnitude of the effects identified by Burton et al, they found that implementing the first strategy yielded a $-\$ 69.77$ reduction in per-beneficiary per-month total spending.

Zazove et $\mathrm{al}^{6}$ focus on a frequently overlooked disability disproportionately affecting the elderly. While the United States Preventive Services Task Force currently grades routine hearing screening in adults an "I", disabling hearing loss affects up to $8 \%$ of adults over 55 , and up to $50 \%$ of adults over $75 .^{7}$ This study uses electronic medical record (EMR) alerts to prompt primary care physicians to screen adults aged 55 years and older for hearing difficulties by asking: "Do you 
have difficulty hearing?" This single question resulted in a fivefold increase in appropriate referrals for further testing vs the control group. During the same time period, Medicare began paying for wellness visits, including hearing screens, which barely affected rates of hearing screens referrals. Hearing loss is a central quality-of-life issue that negatively impacts social interaction, income, cognitive function, quality of life, and risk of depression and increases dependence on social support systems, communication difficulties, hospital admissions, and readmission rates.

Both of these papers highlight a particular strategy for progressing toward the Triple Aim—using tools and registries implemented through an EMR system to improve quality of care-a centerpiece of the PCMH approach. The PCMH model has been widely adopted with the promise of delivering better and more economical, patient-centric care. At a rough estimate, one-third of practicing primary care physicians in the United States are a part of PCMH-recognized practices. ${ }^{8,9}$ However, not all PCMHs or EMR systems are created equal. There are many paths to being recognized as a PCMH that are implemented with varying amounts of rigor. While current federal acts around EMR functionality are starting to get more granular around robust minimum standards for what EMR systems should do, ${ }^{10}$ we are not there yet. It is imperative that the PCMH model continue to evolve toward highyield activities.

The issue here is not that primary care clinics fail to implement best practices, but rather that policy has not made it rational for primary care clinics to implement these proven practices. Obtaining recognition as a PCMH is a cumbersome and costly process, ${ }^{11}$ yet the benefits from PCMHs accrue to the system at large, rather than the clinics bearing the costs. This is a particular challenge for smaller and independent practices. ${ }^{12}$ As the PCMH model evolves, improvements need to include incentives for primary care clinics to implement high yield activities that reduce costs while improving quality of life. Two basic changes that would make it rational for clinics to implement such a model are (1) reimbursement incentives that reflect system cost reductions and quality of life-improving care $_{i}$ and (2) minimum standards for EMR systems that make implementing registries and alerts user friendly, while ensuring the interoperability of EMR systems. If a PCMH model is the best way to capture these successful interventions, then the burden of certification must be reasonable and the costs not a disincentive. As our health care system changes, we must follow the Triple Aim and ensure that we are on a path where affordable care improves the quality of life for patients and practice satisfaction for clinicians.

To read or post commentaries in response to this article, see it online at https://www.AnnFamMed.org/content/18/6/482.

Key words: patient-centered medical home; demonstration; Medicare; spending; utilization; hearing loss; screening; older adults; comorbidity

Submitted September 24, 2020; accepted October 1, 2020.

\section{References}

1. Berwick DM, Nolan TW, Whittington J. The triple aim: care, health, and cost. Health Aff (Millwood). 2008;27(3):759-769. 10.1377/ hlthaff.27.3.759.

2. NHE Fact Sheet. Centers for Medicare and Medicaid Services. Accessed Sep 14, 2020. https://www.cms.gov/Research-StatisticsData-and-Systems/Statistics-Trends-and-Reports/NationalHealth ExpendData/NHE-Fact-Sheet

3. Daniels N. A lifespan approach to health care. In: Jecker NS, ed. Aging And Ethics: Philosophical Problems in Gerontology. Humana Press; 1992:227-246.

4. Daniels N. Just Health: Meeting Health Needs Fairly. Cambridge University Press; 2007.

5. Burton R, Zuckerman S, Haber S, Keyes V. Patient-Centered medical home activities associated with low Medicare spending and utilization. Ann Fam Med. 2020;18(6):503-510.

6. Zazove P, Plegue M, McKee M, et al. Effective hearing loss screening in primary care: the early auditory referral-primary care study (EAR-PC). Ann Fam Med. 2020;18(6):520-527.

7. Hearing loss in older adults: screening. United States Preventive Services Taskforce. Published Aug 15, 2012. Updated Sep 1, 2020. Accessed Sep 19, 2020. https://www.uspreventiveservicestaskforce. org/uspstf/recommendation/hearing-loss-in-older-adults-screening

8. Projecting the Supply and Demand for Primary Care Practitioners Through 2020. US Dept of Health and Human Services. Published Nov 2013. https://bhw.hrsa.gov/sites/default/files/bhw/nchwa/projectingprimarycare.pdf

9. Patient-Centered Medical Home (PCMH). National Committee for Quality Assurance. Accessed Sep 13, 2020. https://www.ncqa.org/ programs/health-care-providers-practices/patient-centered-medicalhome-pcmh/

10. 21st Century Cures Act: Interoperability, Information Blocking, and the ONC Health IT Certification Program. Federal Register. 85 CFR 25642 (2020). https://www.federalregister. gov/documents/2020/05/01/2020-07419/21st-century-cures-actinteroperability-information-blocking-and-the-onc-health-it-certification

11. Bujold $E$. When practice transformation impedes practice improvement. Ann Fam Med. 2015;13(3):273-275.

12. Glancey KK, Kennedy JG. Achieving PCMH status may not be meaningful for small practices. Ann Fam Med. 2016;14(1):4-5. 